\title{
Chiral Thermodynamics in a finite box *
}

\author{
ANA JuRIČIĆ ${ }^{1,2}$ AND BERnd-Jochen SCHAEFER ${ }^{2}$ \\ ${ }^{1}$ Institut für Physik, Universität Graz, Universitätsplatz 5, 8010 Graz, Austria \\ ${ }^{2}$ Institut für Theoretische Physik, Justus-Liebig-Universität Gießen, \\ Heinrich-Buff-Ring 16, 35392 Gießen, Germany
}

\begin{abstract}
Finite-volume modifications of the two-flavor chiral phase diagram are investigated within an effective quark-meson model in various mean-field approximations. The role of vacuum fluctuations and boundary conditions, their influence on higher cumulants and signatures of a possible pseudocritical endpoint are amplified with smaller volumes.
\end{abstract}

\section{Introduction}

At sufficiently high temperature and densities QCD predicts a phase transition from a hadronic phase to a deconfined quark-gluon plasma. Obviously, a plasma created in a laboratory experiment is restricted to a finite (small) volume surrounded by a cold exterior. These volumes are not of a fixed size and one has to deal with ensembles of differently shaped and sized volumes depending on e.g. the centrality of a heavy-ion collision. Thermodynamic quantities directly inferred from such data should not correspond to theoretical predictions obtained in the thermodynamic limit where nonnegligible finite-size corrections are ignored. Furthermore, any system of finite size cannot exhibit a real sharp phase transition and criticality cannot be observed in the usual sense. This regards, in particular, the singularity connected with the existence of a second-order critical endpoint (CEP) in the QCD phase diagram [1]. All critical quantities become pseudocritical with certain ambiguities in their definitions.

The scope of this talk is to address finite-size effects within a well-defined low-energy effective model for the two-flavor chiral phase transition [2]. We employ different approximations of the grand potential to study the influence of quantum and thermal fluctuations on the phase transition in different volumes. Even though the actual obtained numbers in such an effective

* Presented by B.-J. Schaefer at "Critical Point and Onset of Deconfinement" (CPOD2016), Wrocław, Poland; May $30^{\text {th }}-$ June $4^{\text {th }}, 2016$. 
model analysis should not be overrated the generic statements relative to the thermodynamic limit are of relevance which turn out to be of significance. We adopt the two-flavor quark-meson model

$$
\mathcal{L}=\bar{q}\left(i \not \partial-g\left(\sigma+i \vec{\tau} \cdot \vec{\pi} \gamma_{5}\right)+\mu \gamma^{0}\right) q+\frac{1}{2}\left(\partial_{\mu} \phi\right)^{2}-U^{\operatorname{mes}}(\phi)
$$

where the meson fields are conflated in the $O(4)$-symmetric vector $\phi^{T}=$ $(\sigma, \vec{\pi})$ with a purely mesonic potential

$$
U^{\mathrm{mes}}(\phi)=\frac{\lambda}{4}\left(\phi^{2}-v^{2}\right)^{2}-c \sigma .
$$

The four meson fields are coupled to the quark fields $q^{T}=(u, d)$ via a Yukawa coupling $g$. Chiral symmetry is broken explicitly by a linear $\sigma$-term in the potential. The partition function of the model is given by a path integral over all quark and meson fields which cannot be done analytically. In mean-field approximations the path integration is approximated by performing only the quark integration and ignoring the meson integrals. The quark loop yields a generically divergent vacuum contribution to the resulting thermodynamic grand potential. By choosing a regularization scheme with a certain ultraviolet cutoff $\Lambda$ we can investigate the influence of these vacuum fluctuations on the system. In standard mean-field approximation (sMFA) the vacuum term to the potential is simply ignored which corresponds to setting $\Lambda=0$. Since the quark-meson model is a renormalizable model one can fully include the vaccum term by sending $\Lambda \rightarrow \infty$ which we will denote as renormalized mean-field approximation (rMFA) and goes beyond the usual sMFA. In this way vacuum and thermal fluctuations of the quarks are taken into account. In contrast, vacuum and thermal fluctuations of the meson fields are always ignored in such mean-field approximations.

\section{Finite volume effects}

For our investigation of a system in a finite three-dimensional volume we choose a spatial box of equal length $L$. The temporal direction is also compactified to finite size $\beta=1 / T$ to incorporate finite temperature. The boundary conditions in the temperature direction are determined by the spin-statistics theorem and provide the usual thermal Matsubara frequencies for bosons and quarks. In contrast, the boundary conditions in the spatial directions can be chosen independently for bosons and fermions. This freedom is utilized in lattice Monte Carlo simulations where usually periodic boundary conditions for quarks in spatial direction are employed since they ensure a fast approach to the infinite volume limit. Moreover, this enables a further comparison of the influence of the chosen boundary 
conditions on the phase structure. Despite possible generalization to arbitrary phases only periodic $(\mathrm{PBC})$, periodic without the zero mode $\left(\mathrm{PBC}^{*}\right)$ and antiperiodic boundary conditions $(\mathrm{ABC})$ where the zero mode is absent are considered in the following.
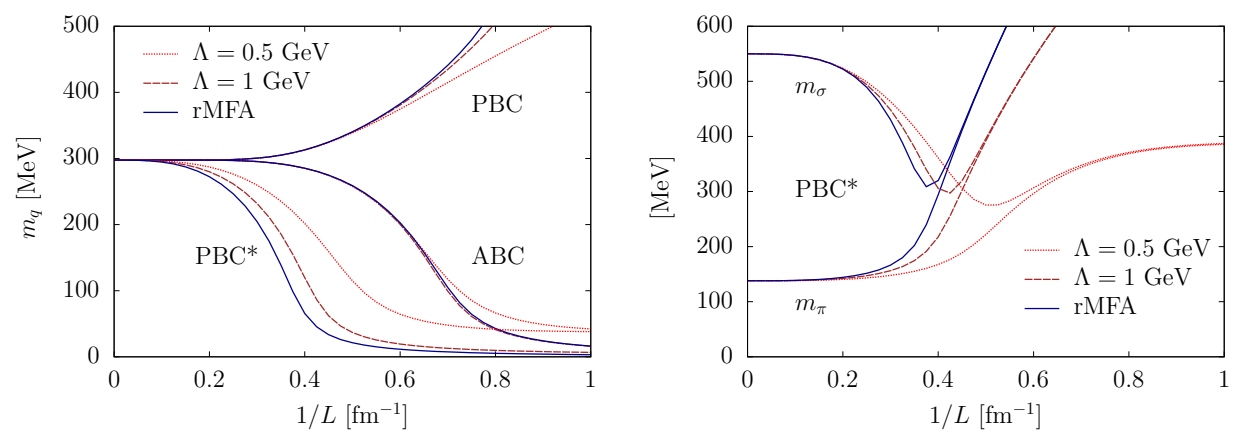

Fig. 1. Quark (left panel) and meson masses (right panel) as a function of the inverse system size for different UV-cutoffs and boundary conditions.

In Fig. 1 the quark masses (left panel) and the curvature meson masses (right panel) are shown as a function of the inverse size. Without the zero mode all masses display a similar behavior as at finite temperature which demonstrates the analogy of the temperature with the inverse size. For decreasing volumes and $\mathrm{PBC}^{*}$ a chiral symmetry restoration "phenomenon" around $1 / L \sim 0.4 \mathrm{fm}^{-1}$ is found which is shifted to smaller volumes for ABC. Moreover, the vacuum fluctuations affect the $L$-dependence stronger in comparison to ABC. This behavior is in contrast to the unphysical PBC case when the zero mode is included and is illustrated in the left panel of Fig. 1.
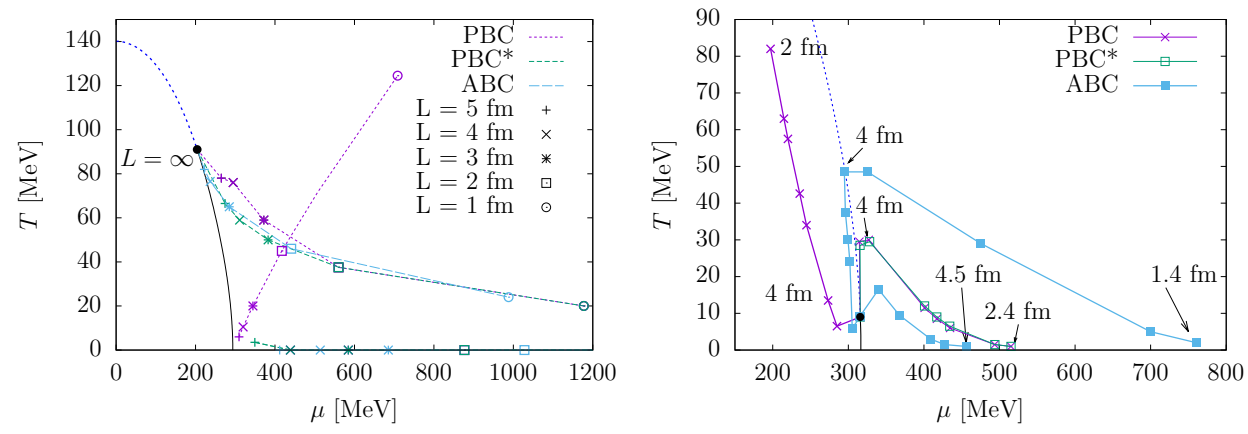

Fig. 2. Size sensitivity of the pseudocritical point for different boundary condition. Left panel without (sMFA) and right panel with vacuum fluctuations (rMFA). 
In Fig. 2 the size-sensitivity of the pseudocritical endpoints for three different spatial boundary conditions are shown and compared to the infinite volume phase diagram obtained in the thermodynamic limit (solid black line represents the first-order transition). The left panel represents the shift of the location without the vacuum fluctuations (sMFA) while these fluctuations are included in the right panel (rMFA). Including the zero mode (PBC) additional discontinuities in the order parameter emerge and as a consequence further endpoints of these discontinuity-lines appear. In addition for small temperatures more discontinuities in the order parameter are visible which we also include in the figure. However, only one endpoint per volume size is connected to the crossover line which we identify as the physical pseudocritical endpoint. Excluding the zero-mode almost no difference between the boundary conditions $(\mathrm{ABC})$ and $\left(\mathrm{PBC}^{*}\right)$ are found. Both boundary conditions reproduce the infinite-volume limit already for sizes $L \geq 10 \mathrm{fm}$ and for smaller volumes the location is pushed to higher chemical potentials. This changes drastically when vacuum fluctuations are taken into account. In rMFA the findings for both boundary conditions coincide for $L \geq 10 \mathrm{fm}$ but start to deviate by finite-size corrections. At first the pseudocritical endpoint moves upwards to higher temperatures and then turns down and vanishes at larger chemical potentials from the phase diagram. If this scenario is a generic one this would be bad news for the current experiments which may not be able to probe this regime. It is also interesting to see finite-size modifications in the crossover temperature at vanishing chemical potential if vacuum fluctuations are considered or not. In sMFA the crossover temperature at $\mu=0$ increases with decreasing system sizes for all used boundary condition strongest for the one without the zero mode $\left(\mathrm{PBC}^{*}\right)$. In rMFA no significant change is seen for the PBC and $\mathrm{ABC}$ in contrast to the one when the zero mode is excluded. For this case the crossover temperature decreases about $20 \%$ for smaller systems and turns back for $L \lesssim 2 \mathrm{fm}$ towards larger values again.

These findings seem to agree with results obtained with the functional renormalization group in a similar quark-meson model truncation where additionally mesonic fluctuations are taken into account. Depending on the pion mass the crossover curvature decreases for decreasing volume sizes for PBC. However, below a certain length scale $m_{\pi} L \lesssim 2$ the curvature turns back and increases again for smaller volumes and even exceeds its infinitevolume value which could be traced back to the spatial zero mode. On the other hand, for $\mathrm{ABC}$ the curvature is a monotonically decreasing function of the volume size. This behavior, in turn, has the consequence that the critical point is pushed towards larger values of the (isospin symmetric) quark chemical potential and smaller temperatures for PBC and sizes in between $2 \lesssim m_{\pi} L<\infty$ [3]. 
In the vicinity of the CEP the scaling behavior can be applied to infer its presence by employing event-by-event analyses in heavy-ion experiments. Higher cumulants of particle multiplicities fluctuations increase with higher powers of the correlation length. Finite-size corrections smooth out such singularities and only a moderate increase is visible. This in particular involves the region where there is a sign change in higher cumulants. In Fig. 3 the negative region of the first three even cumulants are shown in the finite-size phase diagram obtained in rMFA. Around $L \sim 2.4 \mathrm{fm}$ the CEP is gone and the negative region of all cumulants are drastically washed out. Without the vacuum fluctuations the smoothening is more moderate and the negative regions focus around the crossover transition.
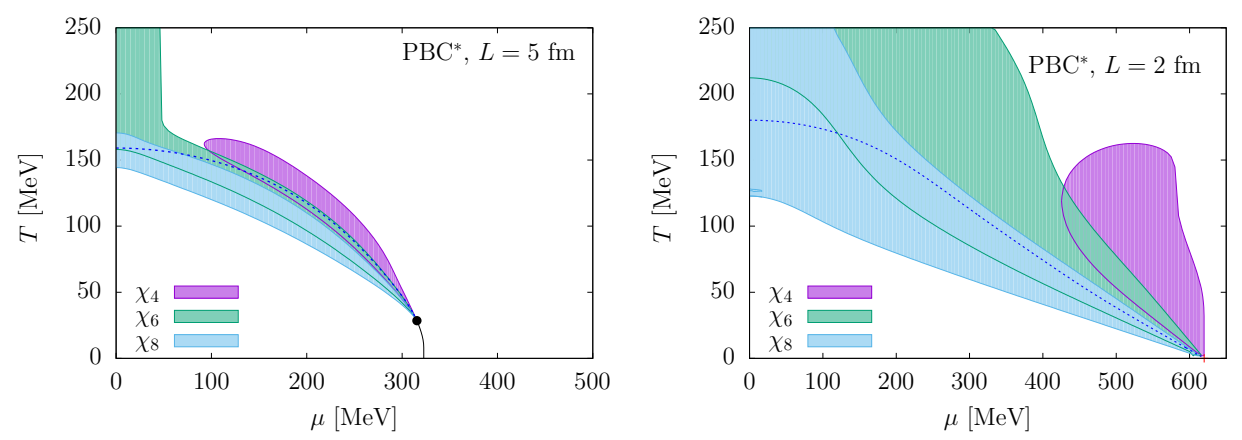

Fig. 3. First three even cumulants $\chi_{4}, \chi_{6}$ and $\chi_{8}$ in the finite-size phase diagram excluding the zero mode in rMFA (left $L=5 \mathrm{fm}$; right $L=2 \mathrm{fm}$ ).

Acknowledgements This work has been supported by the FWF grant P24780N27 and the Helmholtz International Center for FAIR within the LOEWE program of the State of Hesse.

\section{REFERENCES}

[1] J. Braun, B. Klein, and H. Pirner, Phys.Rev. D72, 034017 (2005), hep-ph/ 0504127; Phys.Rev. D71, 014032 (2005), hep-ph/0408116; R.-A. Tripolt, J. Braun, B. Klein, and B.-J. Schaefer, Phys. Rev. D90, 054012 (2014), 1308.0164.

[2] L. F. Palhares, E. S. Fraga, and T. Kodama, J. Phys. G38, 085101 (2011), 0904.4830; E. S. Fraga, L. F. Palhares, and P. Sorensen, Phys. Rev. C84, 011903 (2011).

[3] J. Braun, B. Klein, and B.-J. Schaefer, Phys.Lett. B713, 216 (2012), 1110. 0849. 